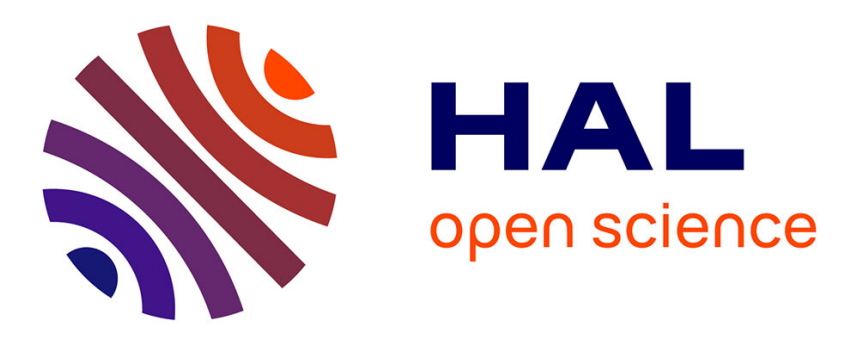

\title{
An Unquantified Uncertainty Visualization Design Space During the Opioid Crisis
}

\author{
Jorin Weatherston, Charles Perin, Dennis K Hore, Bruce Wallace, \\ Margaret-Anne Storey
}

\section{- To cite this version:}

Jorin Weatherston, Charles Perin, Dennis K Hore, Bruce Wallace, Margaret-Anne Storey. An Unquantified Uncertainty Visualization Design Space During the Opioid Crisis. Extended Abstracts of the 2020 CHI Conference on Human Factors in Computing Systems, 2020, Hawai, United States. pp.1-8, 10.1145/3334480.3383072 . hal-02861235

\section{HAL Id: hal-02861235 \\ https://hal.science/hal-02861235}

Submitted on 8 Jun 2020

HAL is a multi-disciplinary open access archive for the deposit and dissemination of scientific research documents, whether they are published or not. The documents may come from teaching and research institutions in France or abroad, or from public or private research centers.
L'archive ouverte pluridisciplinaire HAL, est destinée au dépôt et à la diffusion de documents scientifiques de niveau recherche, publiés ou non, émanant des établissements d'enseignement et de recherche français ou étrangers, des laboratoires publics ou privés. 


\section{An Unquantified Uncertainty Visualization Design Space During the Opioid Crisis}

$\begin{array}{ll}\begin{array}{l}\text { Jorin Weatherston } \\ \text { University of Victoria } \\ \text { Victoria, BC V8P 5C2, Canada } \\ \text { jorinw@uvic.ca }\end{array} & \begin{array}{l}\text { Bruce Wallace } \\ \text { University of Victoria }\end{array} \\ \begin{array}{l}\text { Victoria, BC V8P 5C2, Canada } \\ \text { barclay@uvic.ca }\end{array} \\ \begin{array}{l}\text { Charles Perin } \\ \text { University of Victoria } \\ \text { Victoria, BC V8P 5C2, Canada } \\ \text { cperin@uvic.ca }\end{array} & \begin{array}{l}\text { Margaret-Anne Storey } \\ \text { University of Victoria }\end{array} \\ & \text { mstoria, BC V8P 5C2, Canada } \\ & \end{array}$

Dennis Hore

University of Victoria

Victoria, BC V8P 5C2, Canada

dkhore@uvic.ca

\begin{abstract}
We propose a visualization design space for representing unquantified uncertainty in percent composition drug checking test results using pie and cake charts during the opioid crisis. The design space generates alternatives for use in a visual drug report design study that may improve decisionmaking concerning illicit drug use. Currently, communication of drug checking test results does not capture the uncertainty in drug checking tests, leading to poor and potentially harmful decisions. The design alternatives generated by the design space aim to empower people who use drugs with drug sample information and facilitate harm reduction efforts. Our visualizations may apply to other drug checking services and to scenarios where uncertainty visualization researchers wish to notify end users of the presence of unquantified uncertainty in safety-critical decision-making contexts like those found during the opioid crisis.
\end{abstract}

\section{Author Keywords}

uncertainty, drug-checking, confidence, visualization, decisionmaking

\section{CCS Concepts}

•Human-centered computing $\rightarrow$ Information visualization; 


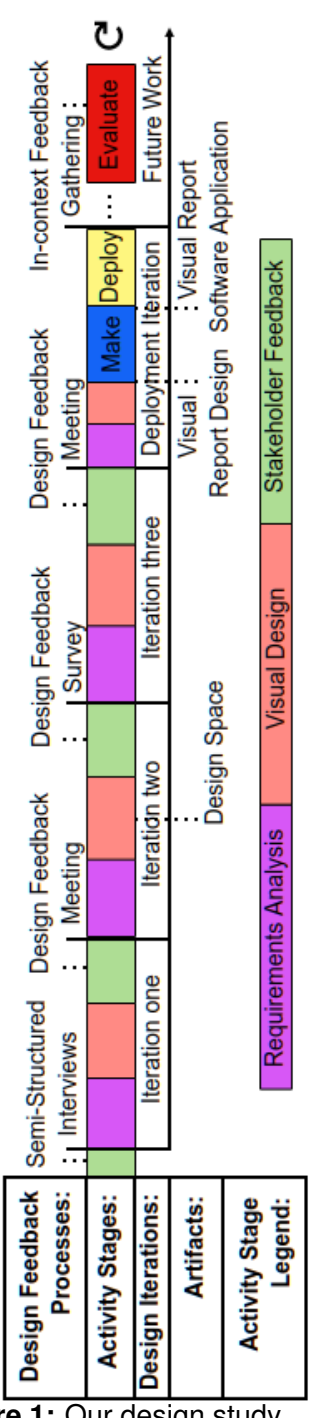

Figure 1: Our design study timeline. The design space was essential in preparing the design feedback survey.

\section{Introduction and Background}

We present a design space for representing unquantified uncertainty in percent composition drug checking test results, where percent composition refers to the proportional contribution of a substance to a drug sample's makeup. We are collaborating with a drug checking service team in Victoria $\mathrm{BC}^{1}$ to produce a visual drug checking test results report (visual report) that supports decision-making by people who access the services, notably people who use drugs. We undertook this research because people who use drugs cannot be certain of drug composition as the illicit drug market is an unregulated market. Opioidsparticularly fentanyl and its potent chemical analogs - have been linked to at least 2,142 deaths between January 2019 and June 2019 , of which $94 \%$ were ruled accidental ${ }^{2}$.

Currently, drug checking test results are presented to service users during in-person conversations with harm reduction and chemical analysis staff in the service. However, misunderstanding percent composition data, especially fentanyl composition, could lead to overdose for drug users.

A global review of drug checking efforts [1] lists eight methods of communicating drug checking test results. Of the reported methods, none we saw a) present uncertainty in their reports, b) expose or resolve discrepancies between tests, or c) provide fentanyl-specific indicators. These three aspects are, however, critical to our collaborators who use five distinct mobile chemical analysis systems to deliver drug sample information.

The work we present in this paper is part of the larger design study research project targeted at generating a comprehensive visual test results report for the drug checking

\footnotetext{
${ }^{1}$ substance.uvic.ca

${ }^{2}$ https://infobase.phac-aspc.gc.ca/datalab/national-surveillance-opioidmortality.html
}

service (see Figure 1). Here we focus on the visualization design space that was crucial in the second iteration of our design study methodology. First we describe the type and extent of the uncertainty being visualized and present a subset of relevant requirements from our design study. We then present the selected proportional charts, describe the design space, and the resulting uncertainty enhanced chart designs. Finally we discuss design space qualities, effectiveness and future research.

\section{Context and Related Work}

In this section we describe contextual information we gathered during the design study and survey of literature.

\section{Data Format and Uncertainty Sources}

The drug checking service uses five types of chemical analyses to generate drug sample data. Percent composition drug sample data is generated by mixture analysis from infrared absorption measurements. Infrared absorption spectra from a sample containing potentially multiple active ingredients and cutting agents are compared to spectra of pure components to determine the identity of the components, and their approximately percent composition. Results of matches are stored as a pair: the name of the substance, and the percent composition (when determined). There are multiple sources of unavoidable uncertainty present in this data type which we show in the sidebar. Despite the challenges faced in visualizing uncertainty, research in uncertainty visualization highlights the risky exclusion, and beneficial inclusion, of uncertainty in datadriven decision making activities [13]. Correll declares that as ethically responsible visualization design researchers, "We ought to visualize hidden uncertainty" [5, p.8]. 


\section{Sources of Uncertainty:}

- Measurement Error: The software bundled with mobile IR absorption systems has limited error reporting abilities, and service turnaround is paramount.

- Manual Subtraction Process: The chemical analysts iteratively subtract components from those identified by the FTIR system. Each component subtraction produces another list which can cause non-determinism in the subsequent components identified.

- Sensitivity: The limit of detection for IR absorption depends on the absorption coefficient of individual molecules in the mixture but is generally around 3\% of weight. Opioids can be dangerous below $3 \%$.

- Incomplete Component Library: The libraries the FTIR depends upon may not possess important chemical signatures of new opioid analoques, limiting the ability to identify them.

\section{Characterizing Uncertainty}

Walker and Marchau describe four levels of uncertainty within decision support systems [17]. Level 1 and 2 are shallow uncertainty, and medium uncertainty, wherein uncertain alternatives are somewhat describe-able. Level 3 and 4 are deep uncertainty, and recognized ignorance, where little to nothing is known of uncertain alternatives.

Potter et al. [11] describe two categories of uncertainty aleatoric uncertainty and epistemic uncertainty. Aleatoric uncertainties are unknowns that arise from statistical variations in measurements. Epistemic uncertainties represents unmitigated unknowns that arise from practical knowledge or measurement limitations. The IR absorption data from our service contains both aleatoric and epistemic uncertainty sources, the combination of which produces level 4 uncertainty, which we name unquantified uncertainty.

Despite the uncertainty in the data being unquantified, providing uncertainty information in drug checking test results is critical in satisfying our safety and ethical requirements.

\section{Visualizing Uncertainty}

Beard and MacKaness's [2, p.40] describe three levels of visual data quality assessments for decision-makers: notification, identification, and quantification. Notification indicates the potential of data problems, identification categorizes the nature of the data quality issue, and quantification shows both the nature and extent of the data problem. In this research we attempt to notify service users of a data problem, as opposed to quantifying a data problem.

During our design study we carefully selected charts to present percent composition data to suit the context and design requirements. Of the charts explored, pie charts display percent composition data effectively and are familiar charts [7]. However, given the controversy of pie charts [15], we also included a complementary alternative chart for stakeholders to choose from during design feedback sessions: the cake chart [3], which is essentially a linearized pie chart. Figure 2 illustrates these two types of charts.

Olston and Mackinlay [10] introduce a technique called ambiguation we adapt to displaying unquantified uncertainty in proportional charts. Combining ambiguation and the application of Bertin's visual variables [4] (and extensions) to uncertainty visualization [9] we conducted design iterations to identify which visual variables we can manipulate to convey unquantified uncertainty to our stakeholders.

\section{Requirements Analysis}

We used an iterative requirements gathering process to generate and evaluate designs with stakeholders as shown in Figure 1. At each iteration we generated what Hevner [6] calls requirements and acceptance criteria: requirements criteria are design cycle inputs used to generate design alternatives, whereas acceptance criteria are design cycle inputs used to measure the effectiveness of the design within the application domain. We kept consolidating these criteria in subsequent design iterations.

The non-functional requirements related to our design goal are a) accessibility to all demographics, b) empowerment of service users with access to underlying data, c) readability of reports with and without service staff, d) transparency of result uncertainty, and, e) usability of the visual report within the harm-reduction conversation and in safety-critical decision-making.

The functional requirements related to our design goal are a) to use only black and white in report design to enable reliable paper copy printing of visual report, b) to show the presence of proportional error despite lack of error quantification, and, c) not to add marks or legends in addition to proportional chart designs. 

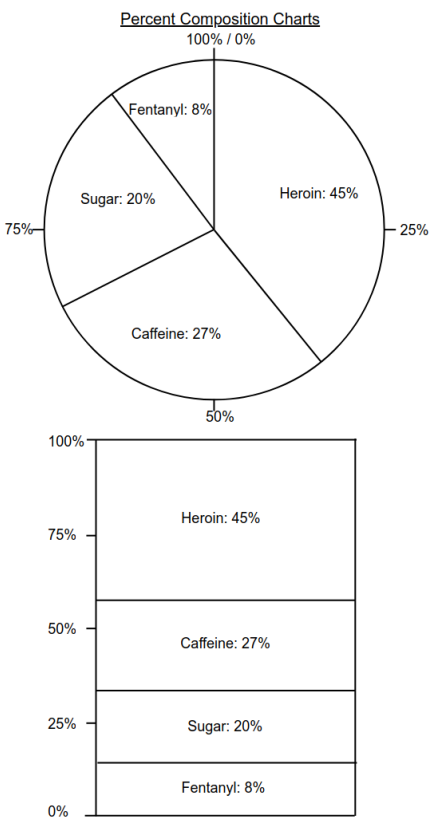

Figure 2: The proportional charts.

The four steps for creating our design space for unquantified uncertainty:

1. Decomposition: Breakdown baseline charts.

2. Dimensions: Outline design space dimensions.

3. Exploration: Explore design space abilities.

4. Application: Apply design space to problem.

5. Evaluation: Evaluate resulting designs.
These requirements describe desirable qualities of valid designs for visualizing the unquantified uncertainty drug testing results data.

\section{An Unquantified Uncertainty Design Space}

For clarity, our use of the design space term is to indicate a dimensionally described space of design possibilities. To lay out the design space of unquantified uncertainty in pie charts and cake charts, we followed the four steps summarized in the sidebar and that we describe in this section.

\subsection{Chart Decomposition}

To visually encode uncertainty in pie and cake charts, we decomposed the charts into six visual marks and their visual variables. We identify six visual marks that both charts share which we show in Figure 3.

- Boundary Edge Marks: The edge between chart segments perpendicular to the percent axis.

- Magnitude Edge Marks: The edge indicating segment size parallel to the percent axis.

- Label Marks: The textual segment labels.

- Areas Marks: The space contained within the boundary and magnitude edge marks.

- Axis Marks: The regularly spaced markings and text parallel to magnitude edge marks used to make segment size comparisons. Ignored in exploration and application stages as they don't present data points.

- Chart Legend: The legend containing pairs of area mark colour and segment information. Also ignored as visually redundant in our colorless application.

\subsection{Design Space Dimensions}

Together visual marks and their visual variables make up the dimensions of the design space. The visual variables control the visual appearance of visual marks, e.g., color,

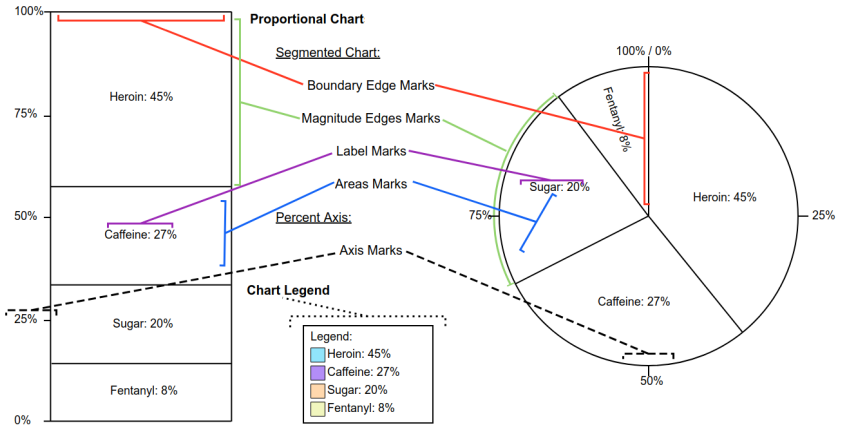

Figure 3: Decomposition of pie and cake chart into visual marks.

width and texture [4]. Visual variables also possess metavariables. A meta-variable we explore later is the extent of manipulation we are applying to the visual variable. The number of dimensions in this design space is the multiplication: visual marks $\times$ visual variables $=D$. A point in the design space is therefore a $D$-length-tuple populated with design choices for each of the design dimensions. To navigate through the design space, one can do so by manipulating the visual variables of each of the visual marks by a chosen degree.

3.0 Exploring Design Concepts

In this step we freely explored and reflected on the design space's abilities without consideration to contextual requirements using the five design sheets methodology [12]. We used visual variables such as color, length, width and pattern to encode uncertainty on each of the visual marks to characterize the balance between encoding uncertainty on separate visual marks and maintaining baseline chart functionality. We show example results of changing the extent of modifications to individual visual variables in Figure 4, with some design concepts working better than others. 


\section{A: LABEL MARK VARIATIONS}

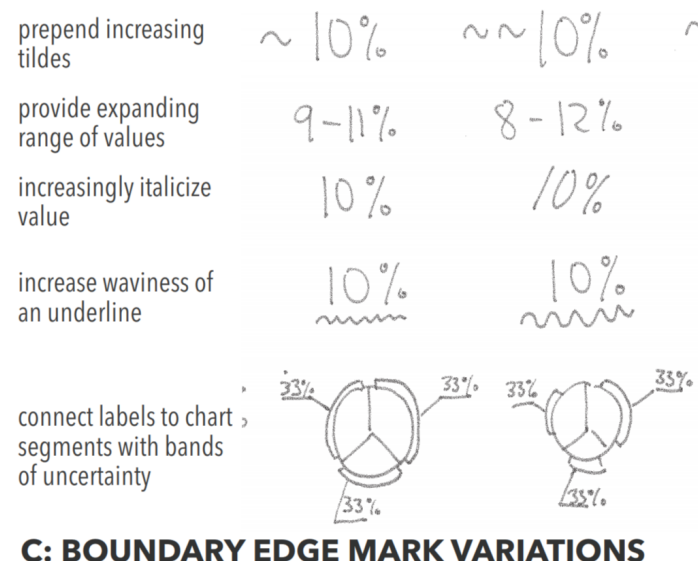

\section{C: BOUNDARY EDGE MARK VARIATIONS}

increase line thickness

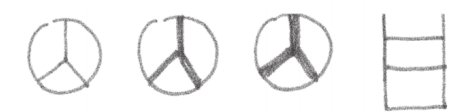

increase line length

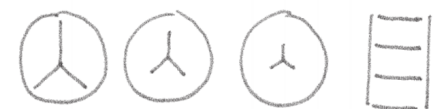

increase line wavines

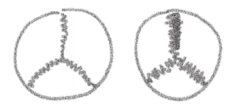

replace solid line with a dotted line and decrease dot density

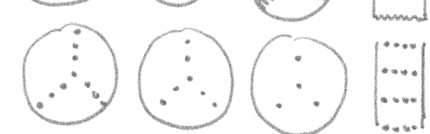

$$
\sim \sim \sim 10 \%
$$

$$
7-13 \%
$$$$
10 \%
$$

$$
10 \%
$$

nun

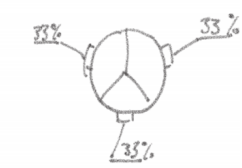
$3 \%$

increase line waviness

replace solid line with

a dotted line and

decrease dot density

\section{B: MAGNITUDE EDGE MARK VARIATIONS}

ncrease line thickness
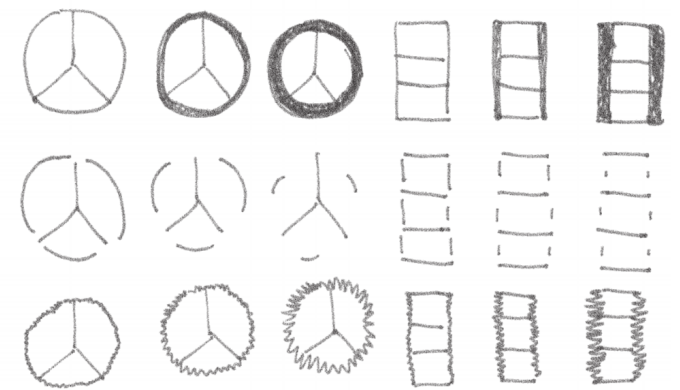

$\therefore$

D: AREA MARK VARIATIONS
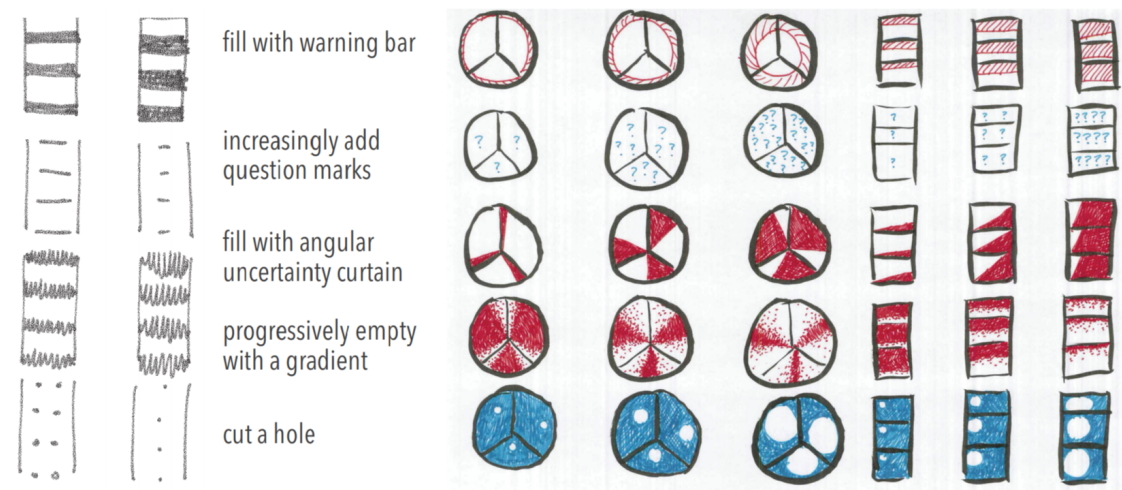

Figure 4: A systematic exploration of the design space to identify which visual marks and visual variables are best suited to notifying service users of the presence of a data problem in the proportional charts. We explore changes to one visual mark in small, medium, and large extents at a time to understand the balance between baseline chart functionality and introducing unquantified uncertainty. 


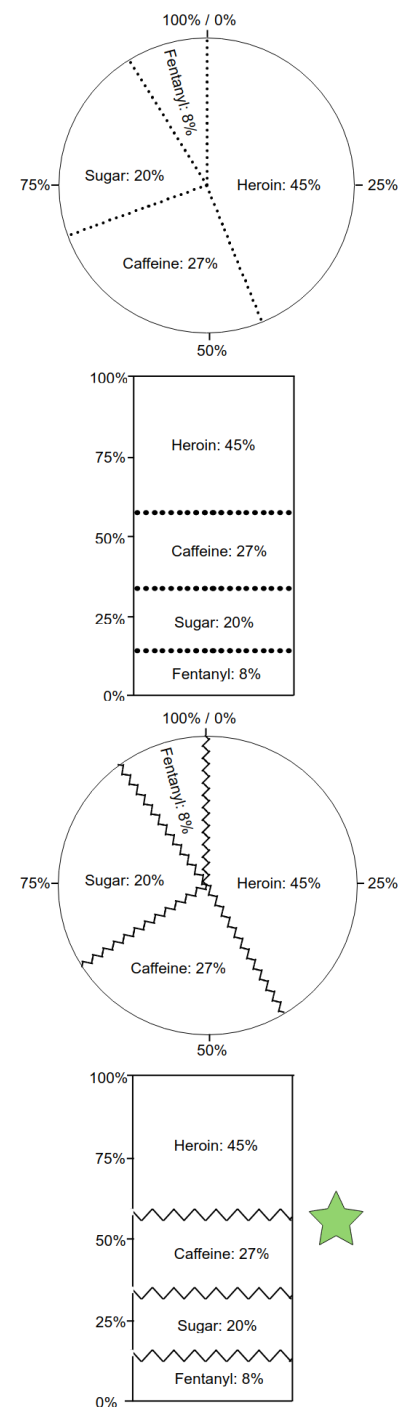

Figure 5: We show examples of produced design alternatives. A green star shows the voted chart.
4.0 Application

We then applied the design space to solve our design challenge of notifying service users of the presence of problems in percent composition data proportions while satisfying our contextual requirements. Notably relevant requirements are using only black and white, not depending on quantified uncertainty values, public accessibility, and readability without service staff assistance.

Though detailing the origin of these requirements is beyond the scope of this paper, they are derived from our stakeholders, context and literature. Unsurprisingly, our collaborators found visual marks well-suited to conveying proportions more intuitive at indicating proportional uncertainty. This concept aligns closely with the "ambiguation" concept introduced by Olston and Mackinlay [10]. We used Skau and Kosara's work [7, 16, 8] indicating that central angles of pie charts are less important than arc lengths and areas in conveying proportion sizes to identify unquantified variations to the line style and width visual variables to the boundary edge visual mark as our best design concept as shown in Figure 5. We translated these pie chart ideas to the cake chart dimensions and conducted a design feedback survey with our service collaborators to finalize design decisions. Our ten collaborators range from 22 to 47 years old and are equally split between men and women. The most popular design was the unquantified uncertainty zig-zag cake chart highlighted with a green star in Figure 5 based on design comments and vote count.

\section{Discussion, Future Work and Conclusion}

Schulz et al. [14] propose to discuss design spaces in terms of completeness and consistency. The completeness of a design space is described by its ability to sufficiently populate its problem space with design solutions [14]. The completeness of our design space is satisfactory as it gen- erates designs that fill all problem spaces we encountered and are accepted as design solutions for our context by our stakeholders. The consistency of a design pace is determined by the frequency of design space points which produce invalid designs (i.e. design instances that violate a basic requirement of the design space) [14]. The consistency of our design space is also satisfactory as widely varying design manipulations along single and multiple simultaneous dimensions generated numerous valid design concepts for use in our design feedback survey.

We agree that pie chart angles are poor indications of seg ment size [16] because of our stakeholders perceiving a limited reduction in baseline functionality of the pie chart with modified boundary edges. However we would like to reinforce this perception with empirical explorations of effective unquantified uncertainty visualizations. We also agree with literature indicating some signification concepts more closely represent uncertainty within data than others [9].

As visualization researchers working within drug checking contexts, we must consider ethical and safety concerns if we are to empower people who use drugs to make informed decisions about their drug use. We hope effective unquantified uncertainty designs generated out of this design space will transfer between drug checking services as parts of visual reports, and to non-drug checking decisionsupport scenarios dealing with unquantified uncertainty.

Our future work involves further iterations in our design study, implementing and deploying selected designs into the visual drug checking test result reports software, and evaluating alternatives in lab and field settings. We would also like to further explore the ethical ramifications of safetycritical decision-making uncertainty visualization. 


\section{REFERENCES}

[1] M J Barratt, M Kowalski, L J Maier, and A Ritter. 2018. Global review of drug checking services operating in 2017. Drug Policy Modelling Program Bulletin 24 (2018).

[2] K. Beard and W. Mackaness. 1993. Visual access to data quality in geographic information systems. Cartographica 30, 2-3 (1993), 37-45. DOI : http://dx.doi.org/10.3138/C205-5885-23M7-0664

[3] B.G. Becker. 1998. Visualizing decision table classifiers. Proc. IEEE Symp. on Information Visualization (1998), 102-105. DOI : http://dx.doi.org/10.1109/infvis. 1998.729565

[4] J. Bertin. 1983. Semiology of graphics. University of Wisconsin Press.

https://books.google.ca/books?id=luZQAAAAMAAJ

[5] Michael Correll and Michael. 2019. Ethical Dimensions of Visualization Research. In Proc. 2019 ACM Conf. on Human Factors in Comput. Syst. ACM Press, New York, New York, USA, 1-13. DOI : http://dx.doi.org/10.1145/3290605.3300418

[6] Alan R Hevner. 2007. A Three Cycle View of Design Science Research. Technical Report 2.

https://aisel.aisnet.org/cgi/viewcontent.cgi? article $=1017$

[7] Robert Kosara and Robert. 2016. An Empire Built On Sand. In Proceedings of the Beyond Time and Errors on Novel Evaluation Methods for Visualization - BELIV '16. ACM Press, New York, New York, USA, 162-168. DOI : http://dx.doi.org/10.1145/2993901.2993909

[8] Robert Kosara and Drew Skau. 2016. Judgment Error in Pie Chart Variations. Eurographics Conf. on
Visualization (2016). DOI :

http://dx.doi.org/10.2312/eurovisshort. 20161167

[9] A. M. MacEachren, R. E. Roth, J. O’Brien, B. Li, D. Swingley, and M. Gahegan. 2012. Visual Semiotics \& Uncertainty Visualization: An Empirical Study. IEEE Trans. Vis. Comput. Graphics 18, 12 (dec 2012), 2496-2505. DOI :

http://dx.doi.org/10.1109/TVCG.2012.279

[10] C. Olston and J.D. Mackinlay. Visualizing data with bounded uncertainty. In IEEE 2002 Symp. on Inf. Vis. IEEE Comput. Soc, 37-40. DOI :

http://dx.doi .org/10.1109/INFVIS . 2002 . 1173145

[11] Kristin Potter, Paul Rosen, and Chris R. Johnson. 2012. From Quantification to Visualization: A

Taxonomy of Uncertainty Visualization Approaches. Springer, Berlin, Heidelberg, 226-249. DOI : http://dx.doi .org/10.1007/978-3-642-32677-6_15

[12] Jonathan C. Roberts, Chris Headleand, and Panagiotis D. Ritsos. 2016. Sketching Designs Using the Five Design-Sheet Methodology. IEEE Trans. Vis. Comput. Graphics 22, 1 (2016), 419-428. DOI : http://dx.doi.org/10.1109/TVCG.2015.2467271

[13] Robert E. Roth. 2009. A Qualitative Approach to Understanding the Role of Geographic Information Uncertainty during Decision Making. Cartogr. Geogr. Inf. Sc. 36, 4 (2009), 315-330. DOI : http://dx.doi.org/10.1559/152304009789786326

[14] Hans Jorg Schulz, Thomas Nocke, Magnus Heitzler, and Heidrun Schumann. 2013. A design space of visualization tasks. IEEE Transactions on Visualization and Computer Graphics 19, 12 (2013), 2366-2375. DOI : http://dx.doi.org/10.1109/TVCG.2013.120 
[15] Harri Siirtola. 2014. Bars, Pies, Doughnuts \& Tables-Visualization of Proportions. In Proceedings of the 28th International BCS Human Computer Interaction Conference ( $\mathrm{HCl} 2014$ ) 28. 240-245.

[16] Drew Skau and Robert Kosara. 2016. Arcs, Angles, or Areas: Individual Data Encodings in Pie and Donut Charts. Comput Graph Forum 35, 3 (2016), 121-130.
DOI : http://dx.doi.org/10.1111/cgf. 12888

[17] J H Walker and W E Marchau. 2010. Classifying and communicating uncertainties in model-based policy analysis. Technical Report 4. 299-315 pages. https://s3. amazonaws. com/academia.edu . documents/44350080/Classifying 